\title{
Introduction to the Special Issue on Affective Computing for Large-scale Heterogeneous Multimedia Data
}

\begin{abstract}
Analyzing human emotional status is of vital significance in human-centered computing, as what people feel directly affects their decision-making. This in turn is beneficial for various downstream applications. For example, policy makers can understand public opinion and consider them; companies can improve their services based on customers' experiences and comments about their products, and outdo their competitors; caregivers can better monitor their patients through mental health assessment. An alternate solution to the direct analysis of traditional physiological responses (e.g., facial expressions and physiological signals) is affective computing (AC) of large-scale user-generated multimedia social data (i.e., text, image, video and audio) that indirectly reflect users' emotions. Affective understanding of large-scale multimedia data (either physiological responses or user-generated data) is very challenging, because it involves understanding human subjective perception as well as behavior. The development of such methods is especially hindered by the affective gap and the subjectivity of emotional perceptions. This special issue (SI) aims to gather technical contributions reporting the most recent progress on affective computing technologies for large-scale heterogeneous multimedia data.
\end{abstract}

This SI received 17 high-quality submissions with authors from all over the world. After rigorous peer review, we accepted seven technical articles and one survey article. The topics, goals, and contributions of these accepted articles are summarized below.

The survey paper "Affective Computing for Large-Scale Heterogeneous Multimedia Data: A Survey," authored mainly by the guest editors, opens this SI. The state-of-the-art AC technologies for various multimedia data are comprehensively reviewed. In addition to the key emotion representation models, the authors summarize and compare the existing datasets, extracted features, machine learning methods, and corresponding results for images, music, videos, and multimodal data. Some challenges and potential directions for future research are also discussed.

The initial two technical articles of this SI focus on affective computing of physiological responses, which are spontaneous components of human emotions. In the article "Characterizing Subtle Facial Movements via Riemannian Manifold," Hong et al. propose to characterize subtle facial movements mainly for micro-expression recognition. To address the challenges of low intensity, transient duration, and small-size data, they design a consolidated framework, MOTion ELucidation (MOTEL), to elucidate hidden movements and provide a hierarchical video representation to characterize the dynamics of facial movements. While facial expressions can be easily suppressed or masked, physiological signals are generally independent of human will, as they are controlled by the sympathetic nervous systems. In the article "Physiological Signals-based Emotion Recognition via High-order Correlation Learning," Zhu et al. propose a novel multihypergraph neural network to model the high-order correlations among multi-modal physiological signals and subjects. Hyperedges are employed to formulate the relations of different vertices, each of which stands for one subject with an involved stimulus. The emotion recognition task is translated into the classification of vertices in the multi-hypergraph structure.

The subsequent three articles investigate new solutions for affective computing of multimedia content, which is increasingly posted online to share people's opinions. The article

(C) 2019 Copyright held by the owner/author(s).

1551-6857/2019/11-ART92

https://doi.org/10.1145/3365845 
"Learning Discriminative Sentiment Representation from Strongly and Weakly Supervised CNNs," by She et al., proposes a multiple kernel network to learn representations from both strongly and weakly supervised CNNs for visual emotion classification. The large-scale data from social images and affective datasets with manual annotation are used to train the weakly supervised and strongly supervised deep models, respectively. Meanwhile, an interesting image emotion dataset, Comics, is constructed and released. The article "A Hierarchical CNN-RNN Approach for Visual Emotion Classification," by Li et al., also studies visual emotion classification. Based on the assumption that emotions are affected by various visual stimuli from low level to high level, they propose a hierarchical CNN-RNN approach to predict emotions based on the fused stimuli by exploiting the dependency among features at different levels. After extracting different levels of features using a dual-CNN network, a stacked bidirectional RNN is employed to model the dependency between these features. Besides the visual content, there are many other factors that influence the perception of emotions, and social community is one of them. In the article "Autonomous Semantic Community Detection via Adaptively Weighted Low-rank Approximation,” Yang et al. propose to automatically detect semantic communities in social networks. A novel nonnegative matrix factorization-based method with an adaptively weighted group-sparse low-rank regularization is developed to determine the number of semantic communities and community structures simultaneously.

The last two articles are about affective computing-based applications. In the article "Soul Dancer: Emotion-Based Human Action Generation," Hou et al. attempt to generate action videos with a specific emotion from a single person image. A two-stage framework is designed, including emotion-based pose sequence generation and target video frame generation. They demonstrate the effectiveness of the framework on their constructed "Soul Dancer" dataset. In the article "Affective Content-aware Adaptation Scheme on QoE Optimization of Adaptive Streaming over HTTP,” Hu et al. propose to optimize the Quality of Experience (QoE) for dynamic adaptive video streaming over HTTP by an affective content-aware adaptation scheme. Considering personal demands to affective content can indeed help to improve the QoE in the commercial video services.

In conclusion, the eight articles contained in this SI cover several important emerging topics of affective computing for large-scale heterogeneous multimedia data. We sincerely hope that these articles can motivate interesting insights and inspirations for the researchers and engineers in the multimedia and affective computing fields. We would like to thank Prof. Alberto Del Bimbo, the Editor-in-Chief of ACM TOMM, and Prof. Stefano Berretti, the Information Director, for their kind support and help of this SI. We also thank the reviewers for their professional and generous efforts in reviewing the submissions, which guarantee the high quality of accepted articles. Finally, we thank all the authors who considered submitting their excellent work to this SI.

Sicheng Zhao

University of California, Berkeley, USA

Dhiraj Joshi

IBM T. J. Watson Research Center, USA

Mohammad Soleymani

University of Southern California, USA

Qiang Ji

Rensselaer Polytechnic Institute, USA

Guest Editors 